\title{
Characterization of the Dynamic Narrowband On-Body to Off-Body Area Channel
}

\author{
David Smith $^{\dagger}$, Leif Hanlen ${ }^{\dagger}$, Jian (Andrew) Zhang ${ }^{\dagger}$,Dino Miniutti ${ }^{\dagger}$, David Rodda, Ben Gilbert \\ National ICT Australia (NICTA) $)^{\ddagger}$ \\ (e-mail: \{David.Smith, Leif.Hanlen, Andrew.Zhang, Dino.Miniutti, David.Rodda, Ben.Gilbert\}@nicta.com.au)
}

\begin{abstract}
A characterization of the dynamic narrowband onbody to off-body area channel is presented based on realtime measurements of the time domain channel response at carrier frequencies near the $900 \mathrm{MHz}$ and $2400 \mathrm{MHz}$ Industrial, Scientific and Medical (ISM) bands. A statistical characterization is presented of received signal amplitude when the subject's body is standing and walking, transmitted from the body to a receiver, $R x$, off the body, with various orientations of the subject's body with respect to the receiver, and various distances from the receiver. Two locations of the transmitter, Tx, on the human body are considered. The Lognormal distribution provides a good fitting model with and without movement. Further, the stability is characterized based on a measure of channel response variance, which is called here the channel variation factor and can characterize the channel coherence time. The on-body to off-body area channel is determined to be generally stable over a period of $25 \mathrm{~ms}$, but the amount of stability is found to be dependent both on movement, $T x$ location on-body, and carrier frequency.
\end{abstract}

\section{INTRODUCTION}

Sensing and actuating devices are becoming sufficiently small to allow multiple sensors to be attached to the body. A network of such sensors around the body is referred to as a (wireless) Body Area Network (BAN). There have been various studies of propagation characteristics for transmission from on the body to another position on the body e.g. [24]. Here we provide results and analysis for a typical bodyarea communications scenario of transmission from on-body to off-body, where the receiver is not located on another body, in contrast with the propagation statistics considered in [5]. Understanding of the statistical characterization and stability of such a scenario is key to future body-area communications implementation.

We characterize the narrowband BAN channel for the $900 \mathrm{MHz}$ and $2400 \mathrm{MHz}$ ISM bands using a wearable antenna as a transmitter, $\mathrm{Tx}$, and a similar antenna receiving, $\mathrm{Rx}$ at an appropriate distance off the body. We considered several distances of $\mathrm{Tx}$ to $\mathrm{Rx}$, several orientations of Tx with respect to Rx, two different positions of Tx on the subject's body, and the case of movement, with subject walking, and the subject

${ }^{\dagger}$ D. Smith, L. Hanlen, D. Miniutti and J. Zhang hold adjunct appointments with the Australian National University.

${ }^{\ddagger}$ National ICT Australia is funded by the Australian Government as represented by the Department of Broadband, Communications and the Digital Economy and the Australian Research Council through the ICT Centre of Excellence program.

A part of this work appeared in [1]. standing. Real-time measurements via a vector signal analyzer (VSA) were performed. Our objective was to answer: -

1) Can the (narrowband) BAN radio channel be characterized using well known statistical fading models for the scenario of transmitting on-body to off-body?

2) What is the dominant statistical model of the BAN channel for off-body communications?

3) What are the characteristics of the temporal stability of the off-body channel over suitable periods?

4) How do the characteristics of temporal stability change with respect to relative position, relative distance, and orientation of $\mathrm{Tx}$ with respect to $\mathrm{Rx}$, as well as the difference in temporal stability between the lower and higher carrier frequencies?

We tested common distributions used to statistically describe fading, the Rayleigh, Normal, Lognormal, Gamma, Weibull and Nakagami-m (which can also approximate Rician distributions). We find that the channel gain can be reasonably modeled by a log-normal distribution. ${ }^{1}$

Temporal stability is quantified by a new parameter, the channel variation factor based on normalized channel gain variance, which is facilitated by real-time VSA measurements of channel response. This factor can also characterize the channel coherence time, and is more suitable than the more common autocorrelation function.

\section{EXPERIMENTAL SETUP}

Wireless on-body to off-body channel measurements were made using a commercial wearable antenna strapped to the body of a $181.5 \mathrm{~cm} / 78 \mathrm{~kg}$ male test subject transmitting to a receiver off-body as follows : -

- The transmit antenna, Tx, was placed at two locations on the test subject: front of chest and right wrist, and the receive antenna, Rx was placed on an aluminum tripod that was fitted with a perspex stand to hold the receive antenna.

- Measurements were taken with a VSA with the test subject standing in four different locations in the room. The horizontal distance between the test subject and $\mathrm{Rx}$ was either 1, 2, 3 or $4 \mathrm{~m}$ at these locations as illustrated in Fig. 1. At each location measurements were taken with the subject facing in four different directions: $0^{\circ}, 90^{\circ}$,

\footnotetext{
${ }^{1}$ Even in the (few) cases where lognormal was not the best fit, it remains
} close according to the Akaike information criterion. 
$180^{\circ}$ and $270^{\circ}$, with $0^{\circ}$ representing the subject facing the receive antenna and $90^{\circ}$ representing the subject facing $90^{\circ}$ to the right of the receive antenna.

- For each orientation and location in the room, measurements were taken with the subject standing still and walking on the spot and the total duration of each measurement was $5 \mathrm{~s}$.

A bit rate of 12.5 Mbps, BPSK and root raised-cosine pulse shaping were chosen. Each channel response was extracted by averaging over a period of $40 \mu$ s by processing 8 PN sequences each with 64 chips. Due to limitations in the receiver hardware, each capture of the eight PN sequences was separated by $2.5 \mathrm{~ms}$ while the data was saved to disk. A wireless system with $1 \mathrm{bit} / \mathrm{s} / \mathrm{Hz}$ modulation efficiency could provide the $10 \mathrm{Mbps}$ required by the 802.15.6 technical requirements document [6] within this bandwidth.

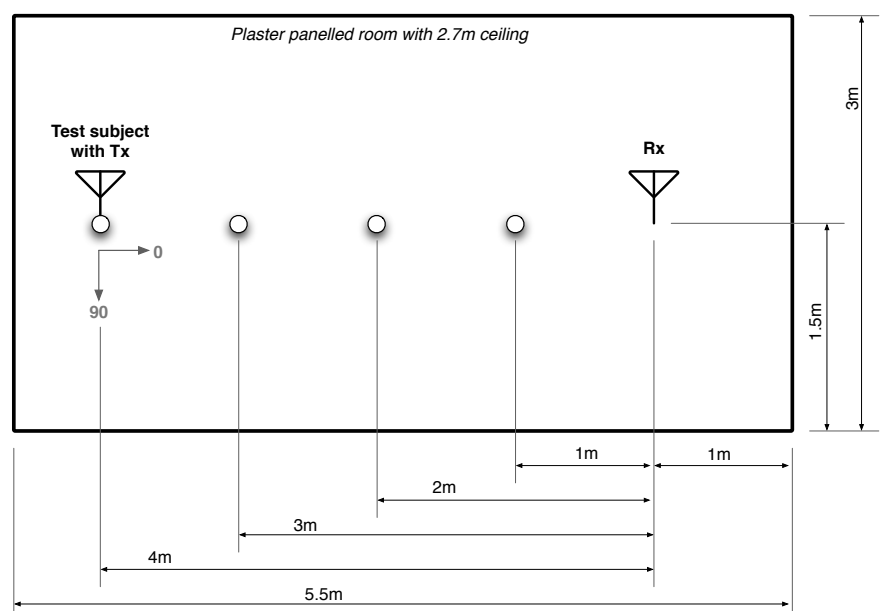

Fig. 1. Experimental environment. Measurements were performed with the test subject $1,2,3$ and 4 meters from the receiver. An angle of $0^{\circ}$ corresponds to the test subject facing the receive antenna.

\section{Statistical Modeling of Received Signal AMPLITUDE DistribUtion}

In this section we attempt to define some reliable statistical models for the signal amplitude distribution over the different scenarios described in the previous section (32 scenarios for Tx on chest, and 32 for Tx on right wrist) with and without movement of the subject.

The measured received power across one set of measurements for a given scenario was normalized according to the maximum received power for that set of measures. The square root of this normalized receive power was taken to find a normalized received signal amplitude.

We obtained maximum likelihood (ML) estimates of received signal amplitude data for these six distributions often used in channel characterization and modeling ${ }^{2}$ : -

\footnotetext{
${ }^{2}$ Due to normalization, distributions are fitted to data that can only take values between 0 and 1 , and we effectively ignore those parts of fitted distribution that takes on values outside this range. The distributions themselves are not normalized.
}

- Normal with probability density function

$$
f(x \mid \mu, \sigma)=\frac{1}{\sigma \sqrt{2 \pi}} \exp \left\{\frac{-(x-\mu)^{2}}{2 \sigma^{2}}\right\} .
$$

- Lognormal

$$
f(x \mid \mu, \sigma)=\frac{1}{x \sigma \sqrt{2 \pi}} \exp \left\{\frac{-(\ln (x)-\mu)^{2}}{2 \sigma^{2}}\right\}
$$

where $\ln (\cdot)$ is the natural logarithm.

- Gamma

$$
f(x \mid a, b)=\frac{1}{b^{a} \Gamma(a)} x^{a-1} \exp \left\{\frac{x}{b}\right\}
$$

where $\Gamma(\cdot)$ is the Gamma function.

- Nakagami-m

$$
f(x \mid m, \omega)=\frac{2 m^{m}}{\Gamma(m)} \frac{x^{2 m-1}}{\omega^{m}} \exp \left\{-\frac{m}{\omega} x^{2}\right\} .
$$

- Weibull

$$
f(x \mid a, b)= \begin{cases}b a^{-b} x^{b-1} \exp \left\{-x / a^{b}\right\} & x \geq 0 \\ 0 & \text { else. }\end{cases}
$$

- Rayleigh

$$
f(x \mid b)=\frac{x}{b^{2}} \exp \left\{\frac{-x}{2 b^{2}}\right\} .
$$

In order to compare between the six distributions we choose the Akaike information criterion (AIC) [7], as used in [8] for wideband characterization, to choose the best fitting distributions for our dynamic narrowband characterization. The second order $\mathrm{AIC}\left(\mathrm{AIC}_{c}\right)$ is given by

$$
\operatorname{AIC}_{c}=-2 \ln (l(\hat{\theta} \mid \text { data }))+2 K+\frac{2 K(K+1)}{(n-K-1)}
$$

where $\ln (l(\hat{\theta} \mid$ data $))$ is the value of the maximized loglikelihood over the unknown parameters $(\theta)$, given the data and the model, $K$ is the number of parameters estimated in the model and $n$ is the sample size. We find the maximized $\log$ likelihood from the ML estimates. The Akaike information criterion can be used as a relative measure, such that the model with the lowest $\mathrm{AIC}_{c}$ approximates the "true" distribution with the minimum loss of information.

We note that from our measurements the sample size $n$ is constant ( $n=2000$ ), and $K=2$ for all distributions apart from the Rayleigh distribution for which $K=1$. Thus in effect we can use the $\mathrm{AIC}_{c}$ to distinguish between a Rayleigh model and the five other models. To compare between the five other models we consider the maximized log-likelihood score.

We stress that in no case does the Rayleigh model provide the best fit for the measured normalized received signal amplitude data across all scenarios at $820 \mathrm{MHz}$ and 2360 $\mathrm{MHz}$ in terms of the $\mathrm{AIC}_{c}$. The distributions, or models, that are the best fitting distributions, according to ML estimates and the subsequent $\mathrm{AIC}_{c}$ are given in Table $\mathrm{I}$ for the case of transmitting from chest and right wrist to $\mathrm{Rx}$ off the body.

Table I shows the best fitting model to the received signal amplitude data, at the lower frequency of $820 \mathrm{MHz}$ and the 
TABLE I

SCENARIOS AND THE DISTRIBUTION (WITH PARAMETERS) THAT GIVES THE BEST OF THE "BEST FITS" TO THE NORMALIZED SIGNAL AMPLITUDE DATA FOR THOSE SCENARIOS TRANSMITTING ON-BODY, TX, TO RX OFF-BODY AT $820 \mathrm{MHZ}$ AND $2.36 \mathrm{GHZ}$ (ANGLE IS ORIENTATION OF THE SUBJECT WITH RESPECT TO THE RECEIVER, D. IS HORIZONTAL DISTANCE FROM SUBJECT TO RECEIVER)

\begin{tabular}{|c|c|c|c|c|c|}
\hline \multirow{2}{*}{ Tx location } & \multirow{2}{*}{ Action } & \multirow{2}{*}{ D. } & \multirow{2}{*}{ Angle } & \multicolumn{2}{|c|}{ Distribution } \\
\hline & & & & $820 \mathrm{MHz}$ & $2.36 \mathrm{GHz}$ \\
\hline Chest & Stand & $1 \mathrm{~m}$ & $0^{\circ}$ & Lognormal $(\mu=-0.0126, \sigma=0.00585)$ & Weibull $(a=0.986, b=121)$ \\
\hline Chest & Stand & $1 \mathrm{~m}$ & $90^{\circ}$ & Weibull $(a=0.988, b=140)$ & Lognormal $(\mu=-0.195, \sigma=0.115)$ \\
\hline Chest & Stand & $1 \mathrm{~m}$ & $180^{\circ}$ & Lognormal $(\mu=-0.135, \sigma=0.0587)$ & Nakagami-m $(m=15.5, \omega=0.692)$ \\
\hline Chest & Stand & $1 \mathrm{~m}$ & $270^{\circ}$ & Weibull $(a=0.958, b=29.4)$ & Normal $(\mu=0.91, \sigma=0.039)$ \\
\hline Chest & Walk & $1 \mathrm{~m}$ & $0^{\circ}$ & Weibull $(a=0.812, b=8.28)$ & Lognormal $(\mu=-0.153, \sigma=0.0592)$ \\
\hline Chest & Walk & $1 \mathrm{~m}$ & $90^{\circ}$ & Gamma $(a=4.31, b=0.085)$ & Nakagami-m $(m=5.7, \omega=0.476)$ \\
\hline Chest & Walk & $1 \mathrm{~m}$ & $180^{\circ}$ & Weibull $(a=0.723, b=5.96)$ & Weibull $(a=0.803, b=7.96)$ \\
\hline Chest & Walk & $1 \mathrm{~m}$ & $270^{\circ}$ & Lognormal $(\mu=-0.688, \sigma=0.287)$ & Nakagami-m $(m=2.04, \omega=0.184)$ \\
\hline Chest & Stand & $2 \mathrm{~m}$ & $0^{\circ}$ & Lognormal $(\mu=-0.071, \sigma=0.0452)$ & Lognormal $(\mu=-0.0255, \sigma=0.00895)$ \\
\hline Chest & Stand & $2 \mathrm{~m}$ & $90^{\circ}$ & Normal $(\mu=0.975, \sigma=0.0111)$ & Lognormal $(\mu=-0.049, \sigma=0.0116)$ \\
\hline Chest & Stand & $2 \mathrm{~m}$ & $180^{\circ}$ & Lognormal $(\mu=-0.069, \sigma=0.0431)$ & Weibull $(a=0.924, b=18)$ \\
\hline Chest & Stand & $2 \mathrm{~m}$ & $270^{\circ}$ & Lognormal $(\mu=-0.0657, \sigma=0.0342)$ & Lognormal $(\mu=-0.171, \sigma=0.0714)$ \\
\hline Chest & Walk & $2 \mathrm{~m}$ & $0^{\circ}$ & Lognormal $(\mu=-0.771, \sigma=0.248)$ & Lognormal $(\mu=-0.207, \sigma=0.0931)$ \\
\hline Chest & Walk & $2 \mathrm{~m}$ & $90^{\circ}$ & Lognormal $(\mu=-0.389, \sigma=0.142)$ & Weibull $(a=0.782, b=4.87)$ \\
\hline Chest & Walk & $2 \mathrm{~m}$ & $180^{\circ}$ & Lognormal $(\mu=-0.905, \sigma=0.361)$ & Gamma $(a=20.9, b=0.0295)$ \\
\hline Chest & Walk & $2 \mathrm{~m}$ & $270^{\circ}$ & Weibull $(a=0.769, b=4.95)$ & Gamma $(a=10.9, b=0.0504)$ \\
\hline Chest & Stand & $3 \mathrm{~m}$ & $0^{\circ}$ & Lognormal $(\mu=-0.156, \sigma=0.0536)$ & Lognormal $(\mu=-0.063, \sigma=0.0153)$ \\
\hline Chest & Stand & $3 \mathrm{~m}$ & $90^{\circ}$ & Weibull $(a=0.974, b=67.3)$ & Weibull $(a=0.966, b=63.8)$ \\
\hline Chest & Stand & $3 \mathrm{~m}$ & $180^{\circ}$ & Lognormal $(\mu=-0.0768, \sigma=0.0409)$ & Lognormal $(\mu=-0.0695, \sigma=0.0223)$ \\
\hline Chest & Stand & $3 \mathrm{~m}$ & $270^{\circ}$ & Lognormal $(\mu=-0.0557, \sigma=0.0251)$ & Lognormal $(\mu=-0.139, \sigma=0.0375)$ \\
\hline Chest & Walk & $3 \mathrm{~m}$ & $0^{\circ}$ & Weibull $(a=0.818, b=7.88)$ & Normal $(\mu=0.8, \sigma=0.0761)$ \\
\hline Chest & Walk & $3 \mathrm{~m}$ & $90^{\circ}$ & Lognormal $(\mu=-0.659, \sigma=0.332)$ & Weibull $(a=0.727, b=4.52)$ \\
\hline Chest & Walk & $3 \mathrm{~m}$ & $180^{\circ}$ & Nakagami-m $(m=2.8, \omega=0.362)$ & Lognormal $(\mu=-0.497, \sigma=0.195)$ \\
\hline Chest & Walk & $3 \mathrm{~m}$ & $270^{\circ}$ & Lognormal $(\mu=-0.713, \sigma=0.381)$ & Weibull $(a=0.859, b=10)$ \\
\hline Chest & Stand & $4 \mathrm{~m}$ & $0^{\circ}$ & Lognormal $(\mu=-0.0361, \sigma=0.0154)$ & Lognormal $(\mu=-0.052, \sigma=0.0157)$ \\
\hline Chest & Stand & $4 \mathrm{~m}$ & $90^{\circ}$ & Lognormal $(\mu=-0.0285, \sigma=0.0156)$ & Lognormal $(\mu=-0.151, \sigma=0.0793)$ \\
\hline Chest & Stand & $4 \mathrm{~m}$ & $180^{\circ}$ & Lognormal $(\mu=-0.0735, \sigma=0.0247)$ & Normal $(\mu=0.947, \sigma=0.0279)$ \\
\hline Chest & Stand & $4 \mathrm{~m}$ & $270^{\circ}$ & Weibull $(a=0.95, b=29.1)$ & Normal $(\mu=0.951, \sigma=0.0193)$ \\
\hline Chest & Walk & $4 \mathrm{~m}$ & $0^{\circ}$ & Normal $(\mu=0.806, \sigma=0.104)$ & Lognormal $(\mu=-0.384, \sigma=0.164)$ \\
\hline Chest & Walk & $4 \mathrm{~m}$ & $90^{\circ}$ & Weibull $(a=0.833, b=6.87)$ & Weibull $(a=0.721, b=4.54)$ \\
\hline Chest & Walk & $4 \mathrm{~m}$ & $180^{\circ}$ & Lognormal $(\mu=-0.625, \sigma=0.327)$ & Weibull $(a=0.732, b=4.74)$ \\
\hline Chest & Walk & $4 \mathrm{~m}$ & $270^{\circ}$ & Lognormal $(\mu=-0.737, \sigma=0.345)$ & Gamma $(a=9.5, b=0.0621)$ \\
\hline Right Wrist & Stand & $1 \mathrm{~m}$ & $0^{\circ}$ & Normal $(\mu=0.972, \sigma=0.0128)$ & Gamma $(a=869, b=0.00106)$ \\
\hline Right Wrist & Stand & $1 \mathrm{~m}$ & $90^{\circ}$ & Normal $(\mu=0.965, \sigma=0.0172)$ & Lognormal $(\mu=-0.0976, \sigma=0.0444)$ \\
\hline Right Wrist & Stand & $1 \mathrm{~m}$ & $180^{\circ}$ & Lognormal $(\mu=-0.0205, \sigma=0.00723)$ & Weibull $(a=0.92, b=17.9)$ \\
\hline Right Wrist & Stand & $1 \mathrm{~m}$ & $270^{\circ}$ & Lognormal $(\mu=-0.0654, \sigma=0.0163)$ & Lognormal $(\mu=-0.0895, \sigma=0.0328)$ \\
\hline Right Wrist & Walk & $1 \mathrm{~m}$ & $0^{\circ}$ & Nakagami-m $(m=0.674, \omega=0.304)$ & Nakagami-m $(m=0.821, \omega=0.213)$ \\
\hline Right Wrist & Walk & $1 \mathrm{~m}$ & $90^{\circ}$ & Lognormal $(\mu=-1.13, \sigma=0.665)$ & Lognormal $(\mu=-1.26, \sigma=0.848)$ \\
\hline Right Wrist & Walk & $1 \mathrm{~m}$ & $180^{\circ}$ & Weibull $(a=0.687, b=3.65)$ & Lognormal $(\mu=-1.58, \sigma=0.751)$ \\
\hline Right Wrist & Walk & $1 \mathrm{~m}$ & $270^{\circ}$ & Weibull $(a=0.645, b=3.3)$ & Gamma $(a=8.06, b=0.0593)$ \\
\hline Right Wrist & Stand & $2 \mathrm{~m}$ & $0^{\circ}$ & Weibull $(a=0.974, b=75)$ & Lognormal $(\mu=-0.106, \sigma=0.0222)$ \\
\hline Right Wrist & Stand & $2 \mathrm{~m}$ & $90^{\circ}$ & Lognormal $(\mu=-0.0493, \sigma=0.014)$ & Lognormal $(\mu=-0.0573, \sigma=0.0237)$ \\
\hline Right Wrist & Stand & $2 \mathrm{~m}$ & $180^{\circ}$ & Lognormal $(\mu=-0.0469, \sigma=0.0192)$ & Gamma $(a=4.66 e+003, b=0.000206)$ \\
\hline Right Wrist & Stand & $2 \mathrm{~m}$ & $270^{\circ}$ & Lognormal $(\mu=-0.0475, \sigma=0.0161)$ & Lognormal $(\mu=-0.0545, \sigma=0.023)$ \\
\hline Right Wrist & Walk & $2 \mathrm{~m}$ & $0^{\circ}$ & Lognormal $(\mu=-0.532, \sigma=0.228)$ & Gamma $(a=3.36, b=0.0978)$ \\
\hline Right Wrist & Walk & $2 \mathrm{~m}$ & $90^{\circ}$ & Lognormal $(\mu=-0.973, \sigma=0.69)$ & Lognormal $(\mu=-1.32, \sigma=0.719)$ \\
\hline Right Wrist & Walk & $2 \mathrm{~m}$ & $180^{\circ}$ & Nakagami-m $(m=2.49, \omega=0.323)$ & Lognormal $(\mu=-1.29, \sigma=0.639)$ \\
\hline Right Wrist & Walk & $2 \mathrm{~m}$ & $270^{\circ}$ & Nakagami-m $(m=2.96, \omega=0.342)$ & Lognormal $(\mu=-0.824, \sigma=0.383)$ \\
\hline Right Wrist & Stand & $3 \mathrm{~m}$ & $0^{\circ}$ & Lognormal $(\mu=-0.0753, \sigma=0.0214)$ & Lognormal $(\mu=-0.255, \sigma=0.124)$ \\
\hline Right Wrist & Stand & $3 \mathrm{~m}$ & $90^{\circ}$ & Lognormal $(\mu=-0.0782, \sigma=0.0289)$ & Weibull $(a=0.938, b=27.6)$ \\
\hline Right Wrist & Stand & $3 \mathrm{~m}$ & $180^{\circ}$ & Normal $(\mu=0.93, \sigma=0.0272)$ & Lognormal $(\mu=-0.0516, \sigma=0.0177)$ \\
\hline Right Wrist & Stand & $3 \mathrm{~m}$ & $270^{\circ}$ & Lognormal $(\mu=-0.0953, \sigma=0.0376)$ & Normal $(\mu=0.96, \sigma=0.0123)$ \\
\hline Right Wrist & Walk & $3 \mathrm{~m}$ & $0^{\circ}$ & Lognormal $(\mu=-1.19, \sigma=0.7)$ & Gamma $(a=3.69, b=0.106)$ \\
\hline Right Wrist & Walk & $3 \mathrm{~m}$ & $90^{\circ}$ & Gamma $(a=4.93, b=0.104)$ & Lognormal $(\mu=-1.19, \sigma=0.606)$ \\
\hline Right Wrist & Walk & $3 \mathrm{~m}$ & $180^{\circ}$ & Weibull $(a=0.677, b=2.75)$ & Lognormal $(\mu=-1.23, \sigma=0.661)$ \\
\hline Right Wrist & Walk & $3 \mathrm{~m}$ & $270^{\circ}$ & Nakagami-m $(m=5.14, \omega=0.437)$ & Lognormal $(\mu=-1.11, \sigma=0.417)$ \\
\hline Right Wrist & Stand & $4 \mathrm{~m}$ & $0^{\circ}$ & Normal $(\mu=0.94, \sigma=0.021)$ & Lognormal $(\mu=-0.0493, \sigma=0.0205)$ \\
\hline Right Wrist & Stand & $4 \mathrm{~m}$ & $90^{\circ}$ & Normal $(\mu=0.941, \sigma=0.0231)$ & Normal $(\mu=0.947, \sigma=0.016)$ \\
\hline Right Wrist & Stand & $4 \mathrm{~m}$ & $180^{\circ}$ & Lognormal $(\mu=-0.0795, \sigma=0.0182)$ & Lognormal $(\mu=-0.0974, \sigma=0.0296)$ \\
\hline Right Wrist & Stand & $4 \mathrm{~m}$ & $270^{\circ}$ & Lognormal $(\mu=-0.118, \sigma=0.032)$ & Normal $(\mu=0.942, \sigma=0.0183)$ \\
\hline Right Wrist & Walk & $4 \mathrm{~m}$ & $0^{\circ}$ & Weibull $(a=0.639, b=3.36)$ & Gamma $(a=6.75, b=0.0691)$ \\
\hline Right Wrist & Walk & $4 \mathrm{~m}$ & $90^{\circ}$ & Weibull $(a=0.644, b=3.08)$ & Lognormal $(\mu=-1.02, \sigma=0.582)$ \\
\hline Right Wrist & Walk & $4 \mathrm{~m}$ & $180^{\circ}$ & Gamma $(a=10.3, b=0.0517)$ & Gamma $(a=6.34, b=0.0737)$ \\
\hline Right Wrist & Walk & $4 \mathrm{~m}$ & $270^{\circ}$ & Weibull $(a=0.656, b=3.41)$ & Lognormal $(\mu=-0.662, \sigma=0.252)$ \\
\hline
\end{tabular}


higher frequency of $2360 \mathrm{MHz}$, is the Lognormal distribution. It also appears that fits are independent of carrier frequency. We also note that the $\mathrm{AIC}_{c}$ is a relative measure and we can confirm that the best fits are good fits empirically by comparing the distribution PDF with the empirical PDF.

In the following figures we show plots of the empirical PDF for the two different positions of Tx location, measurement frequency, and orientation of the subject with respect to Rx. The bin size for the histogram used to describe the PDF from the measured data is chosen according to the "FreedmanDiaconis" rule ${ }^{3}[9]$.

In Fig. 2 an empirical PDF is shown for the case of transmission from the right wrist to $\mathrm{Rx}$ off-body at $2 \mathrm{~m}$ horizontal distance from the body, at $0^{\circ}$ orientation, i.e with subject facing $\mathrm{Rx}$, with the subject walking at the lower measurement frequency of $820 \mathrm{MHz}$ (thus we can expect a line-of-sight (LOS) path). Fig. 3 depicts an empirical PDF for the case of Tx at chest to Rx off-body at $2 \mathrm{~m}$ distance, at $180^{\circ}$ orientation, i.e with subject facing away from $\mathrm{Rx}$, with the subject walking at measured at $820 \mathrm{MHz}$ (thus we can expect no LOS transmission). In Fig. 4 an empirical PDF is shown for the case of transmission from the right wrist to $\mathrm{Rx}$ off-body at $4 \mathrm{~m}$ from the body, at $180^{\circ}$ orientation with the subject standing, at the higher measurement frequency of $2.36 \mathrm{GHz}$, thus we may expect some line-of-sight (LOS) transmission. Overlayed on each figure is the PDF of the best fit for four distributions ${ }^{4}$. In all these cases the Lognormal distribution is the best fit to the measured data, which is the typical result, despite being from quite different scenarios. We also note that the Rayleigh distribution is a very poor fit in these PDF figures, in particular when the subject is standing.

\section{A. Observations}

At this stage, we give the physical explanation for why the Lognormal distribution is in general the best model for our narrowband normalized received signal amplitude characterization. This is the same reason as that shared in [8]. There is a large number of effects contributing to the attenuation of the transmitted signal, such as diffraction, reflection, energy absorption, antenna losses etc. In general these effects are multiplicative, or equivalently additive in the log domain. By the central limit theorem a large number of random multiplicative effects will converge to a normal distribution in the log domain. Due to the office environment, and also for signals propagating from on-body to off-body, there are likely to be additive effects due to combination of multiple paths. It is shown in [10] that adding together Lognormal variables results in a distribution that can be well approximated by another Lognormal distribution.

${ }^{3}$ Bin size $B_{s}$ given by

$$
B_{s}=2 I_{r}(x) n^{-1 / 3}
$$

where $I_{r}$ is the inter-quartile range of the data sample $x$ (in this case measured normalized received power) and $n$ is the sample size of $x$.

${ }^{4}$ Lognormal, Normal, Rayleigh best-fit PDFs are overlayed, with the best of best-fits between Weibull, Gamma and Nakagami-m PDFs also overlayed, which is the Gamma PDF for the cases of the figures shown.

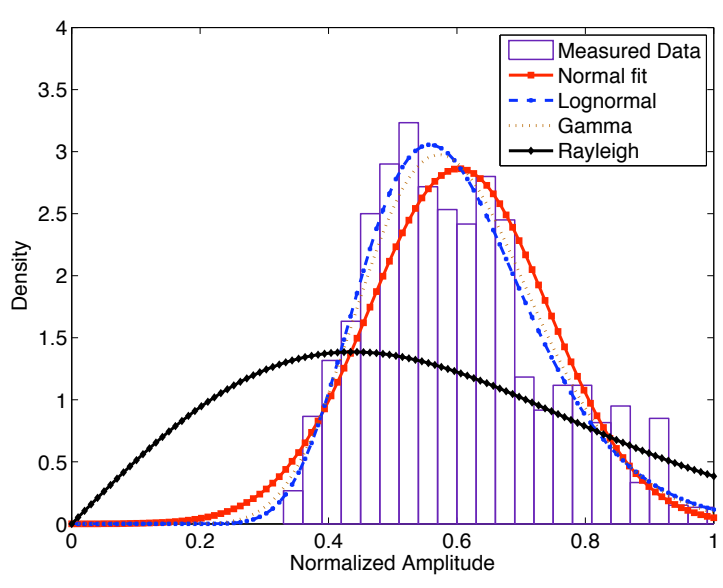

Fig. 2. PDF Right Wrist to Off-Body Rx at $2 \mathrm{~m}, 0^{\circ}$ orientation Rx with respect to Tx, Subject Walking at $820 \mathrm{MHz}$

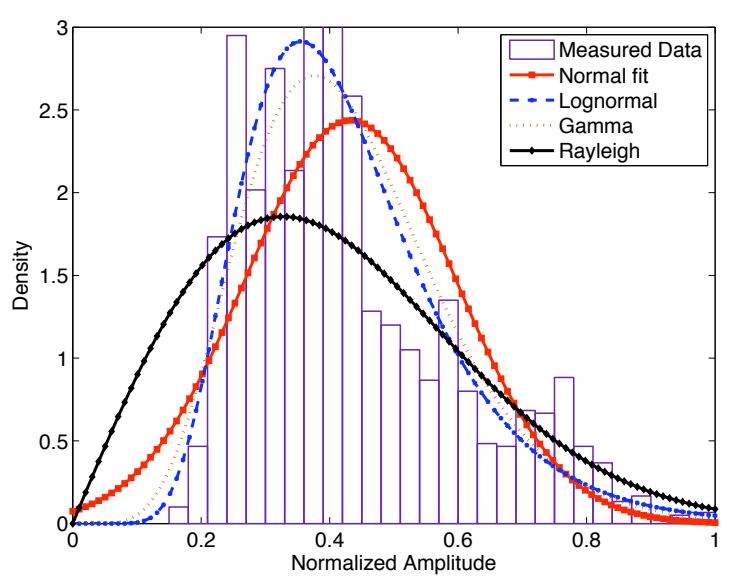

Fig. 3. PDF Chest to Off-Body Rx at $2 \mathrm{~m}, 180^{\circ}$ orientation, Walking at $820 \mathrm{MHz}$

\section{ChanNEL TEMPORAL Stability}

The autocorrelation function is generally used to analyze fading channel variation [11]. The channel coherence time is considered to be the period over which the correlation coefficients are suitably large: the channel is stable within this coherence time. The autocorrelation approach is not suited to characterizing the dynamic WBAN channel because: autocorrelation detects linear dependencies between sequences, not the internal variation within a sequence; and in dynamic WBAN channels a repeated channel response may be observed due to regular movements such as movement of an arm and/or a leg such that autocorrelation analysis will fail to detect the feature of time variation in this case.

We define a new parameter channel variation factor, $\rho$ to characterize WBAN channel stability. The factor is the ratio between the standard deviation (square root of variance) and 


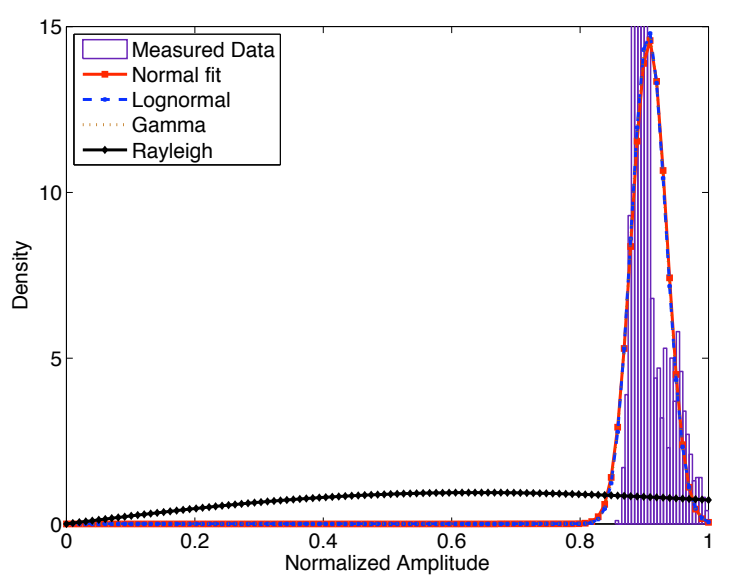

Fig. 4. PDF Right Wrist to Off-Body Rx at $4 \mathrm{~m}, 180^{\circ}$ orientation, Subject Standing at $2360 \mathrm{MHz}$

the root-mean-square power of a channel response sequence ${ }^{5}$. Thus consider channel response sequences of length $M$. For response $m$, we use a vector of $2 L+1=11$ samples $h_{m}(n)$, with $n=-L \ldots L$ aligned so $h_{m}(0)$ is the peak. Thus we compute a channel variation factor for $L$ samples for a span of $\tau=M T_{f}$, where $T_{f}$ is the time interval between two adjacent measured channel responses: 1) Compute the variation factor, $\rho(n)$, for sample $h_{m}(n)$ over $M$ channel responses; 2) Compute the mean over all $L$ samples. The overall channel variation is

$$
\begin{aligned}
\rho(n) & =\sqrt{\frac{\operatorname{var}\left(\left\{\left|h_{k}(n)\right|,\left|h_{k+1}(n)\right|, \cdots,\left|h_{k+M-1}(n)\right|\right\}\right)}{\frac{1}{M} \sum_{m=k}^{M+k-1}\left|h_{m}(n)\right|^{2}}} \\
\rho & =\frac{1}{2 L+1} \sum_{\ell=-L}^{L} \rho(n),
\end{aligned}
$$

where $\operatorname{var}(\cdot)$ represents the variance of a sequence, $k$ is an index used to specify individual channel responses within the set of all measured channel responses, $\rho$ is independent of the received signal power such that $0 \leq \rho \leq 1$.

\section{A. Channel Stability Analysis}

Analysis was conducted with $\tau$ of $5 \mathrm{~ms}, 10 \mathrm{~ms}$ and $25 \mathrm{~ms}$ over a full measurement period of $5 \mathrm{~s}$ in order to characterize onbody to off-body area channel stability. The channel variation factor for all orientations with $\mathrm{Tx}$ at right wrist, with $3 \mathrm{~m}$ separation between $\mathrm{Rx}$ and $\mathrm{Tx}$ and subject walking is shown in Fig. 5.

To better characterize the channel stability, statistical analysis over the channel variation factors was performed on all measurements. We computed empirical cumulative distribution functions (CDFs) from the measured data for the variation

\footnotetext{
${ }^{5}$ We note that there is a discontinuity between measurement sets, which introduces random phase shifts to each channel response. Only the channel magnitude is considered. Furthermore signals from different propagation paths are overlapped and unresolvable causing extended symbol period and waveform distortion
}

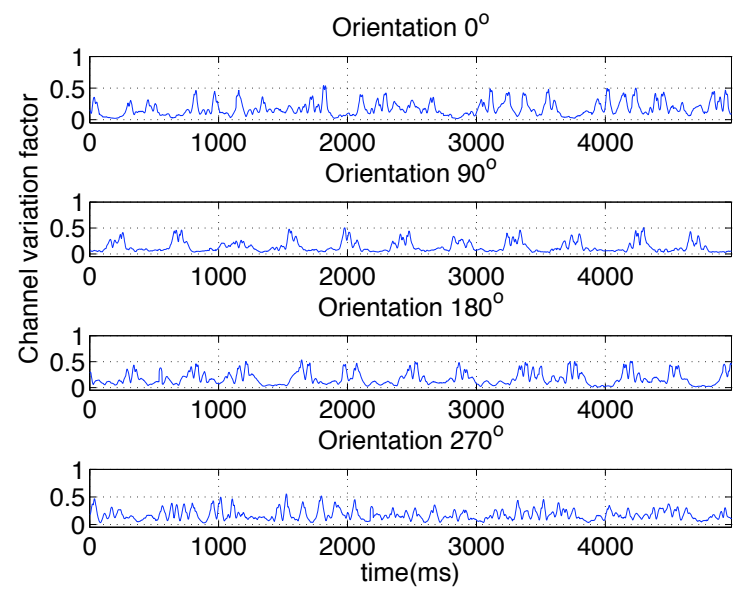

Fig. 5. Channel variation factor for a time varying period of $25 \mathrm{~ms}$, Right wrist to off body, subject walking, Rx Distance $3 \mathrm{~m}$, orientations, $0^{\circ}, 90^{\circ}$, $180^{\circ}$ and $270^{\circ}-2360 \mathrm{MHz}$

factor $\rho$ for all scenarios. Based on these empirical CDFs as a way of describing the stability of the channel, we compute the probability, for a given scenario and time period, that the variation factor is less than 0.1 , or $10 \%$ (Thus the greater the probability the more stable the channel). These probabilities for all scenarios are given in Table II.

Analysis described by empirical CDFs and probabilities in Table II show the following trends: -

- For all scenarios transmitting from the chest there is very good stability in all cases, with marginally greater stability at the lower measurement frequency of $820 \mathrm{MHz}$ than at $2.36 \mathrm{GHz}$.

- Transmitting from the right wrist, the channel is less stable than for transmission from the chest; with far less stability transmitting from the right wrist at $2.36 \mathrm{GHz}$ than for transmitting from the chest at $2.36 \mathrm{GHz}$.

- Furthermore when transmitting from the right wrist the channel is far more stable at $820 \mathrm{MHz}$ than at $2.36 \mathrm{GHz}$.

- When standing there is generally greatest stability with the orientation of the subject's body facing the receiver away from the body (at $0^{\circ}$ ).

- The channel is in generally most stable when the subject is at a distance of $1 \mathrm{~m}$ from the receiver, as opposed to greater distances away from the receiver.

- In general the channel is more stable when the subject is standing than when walking.

- In general it is reasonable to assume channel stability within a $10-25 \mathrm{~ms}$ period.

\section{CONCLUding REMARKS}

A characterization of the dynamic (with and without body movement), human off-body area communications channel has been presented which has application to inform the design of wireless body sensor networks . This characterization was done in proximity to two candidate ISM frequencies of 900 $\mathrm{MHz}$ and $2400 \mathrm{MHz}$. 
TABLE II

PROBABILITIES BASED ON CDFS THAT THE CHANNEL VARIATION FACTOR FOR COHERENCE IS $<0.1$ (OR $10 \%$ ) FOR TIME VARYING PERIODS OF 5MS, 10MS AND 25MS; D-OFF-BOdy RX DistancE From ON-BOdY TX, A-ACTION (S-STANDING, W-WALKING), O-ORIENTATION

\begin{tabular}{|c|c|c|c|c|c|c|c|c|c|c|c|c|c|c|}
\hline \multirow{4}{*}{$\mathrm{D}(\mathrm{m})$} & \multirow{4}{*}{ A } & \multirow{4}{*}{$\mathrm{O}\left({ }^{o}\right)$} & \multicolumn{12}{|c|}{ Probability channel variation factor $<0.1$} \\
\hline & & & \multicolumn{6}{|c|}{ Tx at Chest } & \multicolumn{6}{|c|}{ Tx at Right Wrist } \\
\hline & & & \multicolumn{3}{|c|}{ Periods at $2.36 \mathrm{GHz}$} & \multicolumn{3}{|c|}{ Periods at $820 \mathrm{MHz}$} & \multicolumn{3}{|c|}{ Periods at $2.36 \mathrm{GHz}$} & \multicolumn{3}{|c|}{ Periods at $820 \mathrm{MHz}$} \\
\hline & & & $5 \mathrm{~ms}$ & $10 \mathrm{~ms}$ & $25 \mathrm{~ms}$ & $5 \mathrm{~ms}$ & $10 \mathrm{~ms}$ & $25 \mathrm{~ms}$ & $5 \mathrm{~ms}$ & $10 \mathrm{~ms}$ & $25 \mathrm{~ms}$ & $5 \mathrm{~ms}$ & $10 \mathrm{~ms}$ & $25 \mathrm{~ms}$ \\
\hline 1 & $\bar{S}$ & 0 & 1 & 1 & 1 & 1 & 1 & 1 & 1 & 1 & 1 & 1 & 1 & 1 \\
\hline 1 & $\mathrm{~S}$ & 90 & 0.983 & 1 & 1 & 0.991 & 1 & 1 & 0.969 & 0.993 & 1 & 0.908 & 0.891 & 0.89 \\
\hline 1 & $\mathrm{~S}$ & 180 & 0.977 & 0.96 & 0.933 & 1 & 1 & 1 & 0.968 & 0.987 & 1 & 1 & 1 & 1 \\
\hline 1 & $\mathrm{~S}$ & 270 & 0.969 & 0.998 & 1 & 1 & 1 & 1 & 1 & 1 & 1 & 1 & 1 & 1 \\
\hline 1 & $\mathrm{~W}$ & 0 & 0.998 & 0.997 & 0.997 & 1 & 1 & 1 & 0.891 & 0.73 & 0.384 & 0.99 & 0.981 & 0.927 \\
\hline 1 & $\mathrm{~W}$ & 90 & 0.965 & 0.964 & 0.908 & 0.971 & 0.974 & 0.922 & 0.92 & 0.844 & 0.589 & 0.98 & 0.945 & 0.718 \\
\hline 1 & W & 180 & 0.957 & 0.962 & 0.959 & 0.981 & 0.948 & 0.673 & 0.821 & 0.609 & 0.281 & 0.978 & 0.962 & 0.748 \\
\hline 1 & $\mathrm{~W}$ & 270 & 0.969 & 0.969 & 0.959 & 0.984 & 0.974 & 0.867 & 0.831 & 0.642 & 0.352 & 0.969 & 0.92 & 0.753 \\
\hline 2 & $\mathrm{~S}$ & 0 & 0.998 & 0.997 & 0.997 & 1 & 1 & 1 & 0.92 & 0.952 & 0.99 & 1 & 1 & 1 \\
\hline 2 & $\mathrm{~S}$ & 90 & 0.965 & 0.964 & 0.908 & 0.971 & 0.974 & 0.922 & 0.842 & 0.806 & 0.846 & 0.995 & 1 & 1 \\
\hline 2 & $\mathrm{~S}$ & 180 & 0.957 & 0.962 & 0.959 & 0.981 & 0.948 & 0.673 & 0.878 & 0.886 & 0.933 & 1 & 1 & 1 \\
\hline 2 & S & 270 & 0.969 & 0.969 & 0.959 & 0.984 & 0.974 & 0.867 & 0.888 & 0.932 & 0.979 & 0.999 & 1 & 1 \\
\hline 2 & W & 0 & 0.999 & 1 & 1 & 1 & 1 & 1 & 0.853 & 0.649 & 0.334 & 0.97 & 0.961 & 0.847 \\
\hline 2 & $\mathrm{~W}$ & 90 & 0.977 & 0.999 & 1 & 1 & 1 & 1 & 0.841 & 0.646 & 0.414 & 0.964 & 0.924 & 0.687 \\
\hline 2 & W & 180 & 0.968 & 0.986 & 0.998 & 1 & 1 & 1 & 0.829 & 0.624 & 0.287 & 0.968 & 0.935 & 0.742 \\
\hline 2 & $\mathrm{~W}$ & 270 & 1 & 1 & 1 & 1 & 1 & 1 & 0.888 & 0.727 & 0.283 & 0.981 & 0.962 & 0.736 \\
\hline 3 & $\mathrm{~S}$ & 0 & 0.999 & 1 & 1 & 1 & 1 & 1 & 0.942 & 0.936 & 0.964 & 0.989 & 0.991 & 0.993 \\
\hline 3 & $\mathrm{~S}$ & 90 & 0.977 & 0.999 & 1 & 1 & 1 & 1 & 0.642 & 0.467 & 0.282 & 0.999 & 1 & 1 \\
\hline 3 & S & 180 & 0.968 & 0.986 & 0.998 & 1 & 1 & 1 & 0.791 & 0.737 & 0.672 & 0.869 & 0.905 & 0.94 \\
\hline 3 & $\mathrm{~S}$ & 270 & 1 & 1 & 1 & 1 & 1 & 1 & 0.986 & 1 & 1 & 0.995 & 1 & 1 \\
\hline 3 & W & 0 & 0.988 & 0.985 & 0.978 & 0.988 & 0.984 & 0.979 & 0.791 & 0.59 & 0.264 & 0.962 & 0.934 & 0.639 \\
\hline 3 & W & 90 & 0.983 & 0.992 & 0.955 & 0.977 & 0.972 & 0.977 & 0.848 & 0.728 & 0.539 & 0.968 & 0.914 & 0.65 \\
\hline 3 & W & 180 & 0.978 & 0.974 & 0.964 & 0.963 & 0.917 & 0.719 & 0.806 & 0.625 & 0.345 & 0.904 & 0.795 & 0.488 \\
\hline 3 & W & 270 & 0.978 & 0.97 & 0.897 & 0.983 & 0.963 & 0.818 & 0.807 & 0.573 & 0.201 & 0.978 & 0.957 & 0.797 \\
\hline 4 & $\mathrm{~S}$ & 0 & 0.988 & 0.985 & 0.978 & 0.988 & 0.984 & 0.979 & 1 & 1 & 1 & 1 & 1 & 1 \\
\hline 4 & S & 90 & 0.983 & 0.992 & 0.955 & 0.977 & 0.972 & 0.977 & 0.586 & 0.367 & 0.156 & 1 & 1 & 1 \\
\hline 4 & $\mathrm{~S}$ & 180 & 0.978 & 0.974 & 0.964 & 0.963 & 0.917 & 0.719 & 0.745 & 0.653 & 0.549 & 0.99 & 1 & 1 \\
\hline 4 & $\mathrm{~S}$ & 270 & 0.978 & 0.97 & 0.897 & 0.983 & 0.963 & 0.818 & 0.754 & 0.695 & 0.64 & 0.976 & 0.989 & 0.986 \\
\hline 4 & $\mathrm{~W}$ & 0 & 1 & 1 & 1 & 1 & 1 & 1 & 0.849 & 0.68 & 0.271 & 0.97 & 0.957 & 0.746 \\
\hline 4 & W & 90 & 0.969 & 0.993 & 1 & 0.908 & 0.891 & 0.89 & 0.879 & 0.788 & 0.501 & 0.939 & 0.879 & 0.722 \\
\hline 4 & $\mathrm{~W}$ & 180 & 0.968 & 0.987 & 1 & 1 & 1 & 1 & 0.903 & 0.769 & 0.405 & 0.957 & 0.932 & 0.757 \\
\hline 4 & W & 270 & 1 & 1 & 1 & 1 & 1 & 1 & 0.832 & 0.657 & 0.317 & 0.937 & 0.869 & 0.57 \\
\hline
\end{tabular}

It is clear that the signal amplitude distribution for various movement is typically poorly described by the Rayleigh distribution. This can be seen graphically from probability density functions (PDFs) compared to empirical PDFs, and is demonstrated by derivation of the Akaike information criterion. In some cases the Weibull, Nakagami-m and Gamma distributions provide good fits to the normalized amplitude distribution according to their maximum-likelihood (ML) estimates. However in general the Lognormal distribution provides best fit to the received signal statistics.

It is also clear that the on-body to off-body narrowband WBAN channel is quite stable, and more so at $820 \mathrm{MHz}$ than $2360 \mathrm{MHz}$. A coherence time of $25 \mathrm{~ms}$ is a reasonable assumption for most scenarios. It is also clear that on-body Tx position and carrier frequency are important considerations for channel temporal stability.

\section{REFERENCES}

[1] D. Miniutti, L. Hanlen, D. Smith, A. Zhang, D. Lewis, D. Rodda, and B. Gilbert, "Narrowband On-Body to Off-Body Channel Characterization for Body Area Networks," 15-08-0033-02-0006-draft-of-channelmodel-for-body-area-network.

[2] A. Fort, C. Desset, P. Wambacq, and L. Biesen, "Indoor body-area channel model for narrowband communications," Microwaves, Antennas \& Propagation, IET, vol. 1, no. 6, pp. 1197-1203, Dec. 2007.
[3] S. Obayashi and J. Zander, "A body-shadowing model for indoor radio communication environments," Antennas and Propagation, IEEE Transactions on, vol. 46, no. 6, pp. 920-927, Jun 1998.

[4] P. Hall, Y. Hao, Y. Nechayev, A. Alomalny, C. Constantinou, C. Parini, M. Kamarudin, T. Salim, D. Hee, R. Dubrovka, A. Owadally, W. Song, A. Serra, P. Nepa, M. Gallo, and M. Bozzetti, "Antennas and propagation for on-body communication systems," Antennas and Propagation Magazine, IEEE, vol. 49, no. 3, pp. 41-58, June 2007.

[5] S. Cotton, W. Scanlon, and J. Guy, "The $\kappa-\mu$ distribution applied to the analysis of fading in body to body communication channels for fire and rescue personnel," Antennas and Wireless Propagation Letters, IEEE, vol. 7, pp. 66-69, 2008.

[6] B. Zhen, M. Patel, S. Lee, and E. Won, "Body Area Network (BAN) Technical Requirements,” 15-08-0037-01-0006-ieee-802-15-6-technicalrequirements-document-v-4-0.

[7] H. Akaike, "Information theory as an extension of the maximum likelihood principle," in Proc. 2nd. Int. Inf. Theory Syst., 1973, pp. 267281

[8] A. Fort, C. Desset, P. deDoncker, P. Wambacq, and L. van Biesen, "An ultra-wideband body area propagation channel model-from statistics to implementation," IEEE Transactions on Microwave Theory and Techniques, vol. 54, no. 4, pp. 1820-1826, April 2006.

[9] D. Freedman and P. Diaconis, "On the histogram as a density estimator: $L_{2}$ theory," Probability Theory and Related Fields, vol. 57, no. 4, pp. 453-476, December 1981

[10] L. Fenton, "The sum of log-normal probability distributions in scatter transmission systems," IEEE Trans. Commun. Syst., vol. CS-8, no. 1, pp. 57-67, June 1960.

[11] H. L. Bertoni, Radio Propagation for Modern Wireless Systems. Upper Saddle River, NJ: Prentice Hall, 2000. 